\title{
Reduction of ciliary beat frequency in vitro by sputum from patients with bronchiectasis: a serine proteinase effect
}

\author{
LA SMALLMAN, SL HILL, RA STOCKLEY \\ From the Department of Pathology, Medical School, University of Birmingham, and General Hospital, \\ Birmingham
}

ABSTRACT We have examined the effect of adding elastase positive sputum from six patients with purulent bronchiectasis on the ciliary beat frequency of nasal epithelium from normal subjects. Control studies of cilia suspended in tissue culture medium showed little change in ciliary beat frequency over six hours. Cilia incubated in elastase positive secretions, however, showed a considerable decrease in ciliary beat frequency over the period, falling from a mean of 13.40 beats/second to 6.78 beats/second $(p<0.001)$. Inhibition of the elastase activity with pure human $\alpha_{1}$ antitrypsin abolished this effect (mean at start 13.75 beats/second, mean at six hours 11.64 beats/second). The patients were then treated with amoxycillin for two weeks and sputum was collected at the end of the course. These secretions showed no detectable elastase activity and also had little effect on ciliary beat frequency. The results suggest that serine proteinase activity associated with elastase can decrease mucociliary function in vitro and that antibiotic treatment even in the apparently stable state may have a beneficial effect.

There is a well recognised association between ciliary dysfunction and human disease. The "immotile cilia syndrome" or "primary ciliary dyskinesia" is a hereditary condition in which there is little or no mucociliary transport. The cilia are immotile or dyskinetic and have abnormal ultrastructure. The phenomenon is known to be associated with sinusitis, bronchiectasis, and male infertility. ${ }^{12}$ Other causes of ciliary dysfunction have, however, been reported, including identification of ciliary dyskinetic factors found in the serum of patients with cystic fibrosis, ${ }^{3}$ various respiratory and autoimmune diseases, ${ }^{4}$ and asthma. ${ }^{5}$ Dulfano et $a^{6}{ }^{67}$ have shown that sputum from some asthmatic patients is also capable of producing ciliary inhibition when incubated with frog palate mucosa and human bronchial mucosa.

Recent in vitro data have shown that leucocyte elastase (a proteolytic enzyme from the neutrophil polymorph) is capable of inhibiting ciliary activity and damaging respiratory epithelium. ${ }^{8}$ The secre-

Address for reprint requests: Dr RA Stockley, General Hospital, Birmingham B4 6NH.

Accepted 9 May 1984 tions of patients with purulent bronchiectasis usually show the presence of free elastase activity; ${ }^{9}$ these patients often have evidence of both reduced mucociliary clearance ${ }^{10}$ and epithelial damage. ${ }^{11}$

The present study was initially undertaken to determine the effect of elastase positive bronchial secretions from patients with purulent bronchiectasis on ciliary beat frequency in order to discover whether this was similar to that of the pure enzyme. With these basic data we wished to determine whether inhibition of the elastase activity by pure human $\alpha$ antitrypsin or removal of the elastase activity after antibiotic treatment altered the effect of the secretions on ciliary beat frequency.

\section{Methods}

Nasal cilia were obtained from healthy people by a non-invasive technique similar to that described by Rutland and colleagues. ${ }^{12}{ }^{13}$ Cellular material adhering to a cytology brush (Meditech $1.73 \mathrm{~mm}$ diameter) was dislodged by brisk agitation in $2 \mathrm{ml}$ of tissue culture medium (Medium 199 with Earle's salts and Hepes, Flow Laboratories).

Fragments of nasal epithelium were transferred from the tissue culture medium with a Pasteur 
pipette and deposited on a welled microscope slide $(26 \times 45 \mathrm{~mm})$. A coverslip was gently placed over the microscope slide and the edges were sealed with a mixture of molten petroleum jelly and paraffin wax.

The ciliary beat frequency was measured photometrically. The slide was placed on an electronically controlled warm stage maintained at $37^{\circ} \mathrm{C}$, mounted on a Zeiss Universal microscope. A Zeiss SF photometer attached to the head of the microscope transduced light intensity into an electrical signal. Beating cilia were easily seen at a magnification of $\times 500$ by bright field illumination, positioned so that they intermittently obstructed the passage of light through a small diaphragm $\left(3.14 \mu^{2}\right)$ into the photometer. The transduced electrical signal was amplified and displayed on an oscilloscope screen (Tektronix 564B). Black and white photographs of the traces were then taken to provide a permanent record of ciliary activity and these were analysed later.

Preliminary studies were carried out to assess the stability of ciliary beat frequency and to determine how many areas needed to be counted to provide reproducible results. Four preparations were made from the same sample and ciliary beat frequency was measured in three, six, and 10 areas. The mean and standard error of the mean (SEM) were then calculated. These initial results are summarised in table 1 and although the results are generally comparable it was decided to determine the mean of 10 areas for all subsequent studies.

\section{SAMPLES FROM PATIENTS}

Eight patients with a diagnosis of purulent bronchiectasis confirmed by bronchography were studied during the clinically well state in the absence ot any symptoms suggestive of an acute exacerbation. Sputum was collected over four hours from each patient in a sterile pot as free from saliva as possible. The samples were ultracentrifuged at $50000 \mathrm{~g}$ for 90 minutes $\left(3^{\circ} \mathrm{C}\right)$ to obtain the sol phase; the gel phase was discarded. The presence of elastase activity in the samples was confirmed by elastin agarose diffusion plates (lower limit of detection equivalent

Table 1 Ciliary beat frequency of four slide preparations of human nasal epithelium measured from three, six and 10 areas (values are means with standard errors in parentheses)

\begin{tabular}{llll}
\hline $\begin{array}{l}\text { Slide } \\
\text { preparation }\end{array}$ & \multicolumn{3}{l}{ No of areas } \\
\cline { 2 - 4 } & 3 & 6 & 10 \\
\hline 1 & $14.34(1.08)$ & $14.24(0.70)$ & $14.00(0.53)$ \\
2 & $13.12(1.30)$ & $13.47(0.71)$ & $13.97(0.48)$ \\
3 & $13.47(1.01)$ & $13.43(0.57)$ & $13.58(0.36)$ \\
4 & $13.97(1.09)$ & $13.98(0.72)$ & $14.08(0.53)$ \\
\hline
\end{tabular}

to $0.8 \mu \mathrm{g}$ porcine pancreatic elastase $/ 100 \mu \mathrm{l})$. The $\mathrm{pH}$ of all the samples was measured with a PyeUnicam $9418 \mathrm{pH}$ meter with a surface electrode.

The patients were then treated with amoxycillin ( $250 \mathrm{mg}$ three times daily) for two weeks and repeat samples of sputum were collected on the last day of treatment and examined. In six patients the sputum cleared from purulent to mucoid and elastase activity was lost; secretions from these patients were used for the ciliary beat frequency studies.

\section{EFFECT ON CILIARY BEAT FREQUENCY}

Tissue culture medium within the welled microscope slide was aspirated by a Pasteur pipette and filter paper and the well flooded with the secretion to be analysed $(200 \mu \mathrm{l})$. The slide was then sealed and ciliary beat frequency measured hourly for six hours. It was kept in a humidified chamber at $37^{\circ} \mathrm{C}$ between measurements.

\section{INHIBITION STUDIES}

Alpha ${ }_{1}$ antitrypsin was purified from human serum as described previously ${ }^{14}$ and dissolved in phosphate buffered saline (PBS), pH 7.2. The experiments were then repeated after sufficient $\alpha$ antitrypsin had been added to inhibit the elastase activity (confirmed by the elastin plate) in the purulent secretions. As this usually required a 1:2 dilution of the secretions, experiments were also performed with the same amount of PBS alone added to the secretions to act as a control for any dilutional effect that the $\alpha$ antitrypsin PBS preparation might have on the ciliary beat frequency.

The significance of changes in ciliary beat frequency due to the secretions and the effect of $\alpha_{1}$ antitrypsin and antibiotic treatment were determined with Student's $t$ test.

\section{Results}

The four slide preparations showed that cilia continued to beat well for up to six hours without change of the tissue culture medium, although there was a slow decline in ciliary beat frequency during this period (table 2).

The $\mathrm{pH}$ range of the sputum samples was 6.7-9.45.

The purulent secretions containing elastase activity from the patients with bronchiectasis showed an inhibitory effect on ciliary beat frequency, with a decrease from 13.40 (SEM 0.23 ) beats/s to 6.78 $(0 \cdot 27)$ beats/s over six hours. The change was significantly greater $(p<0.001)$ than the reduction in ciliary beat frequency seen in tissue culture medium alone from one hour onwards (figs 1 and 3).

Inhibition of the elastase activity of the secretions 
Table 2 Ciliary beat frequency of four slide preparations of human nasal epithelium suspended in tissue culture medium for six hours (values are means with standard errors in parentheses)

\begin{tabular}{|c|c|c|c|c|c|c|c|}
\hline \multirow{2}{*}{$\begin{array}{l}\text { Slide } \\
\text { preparation }\end{array}$} & \multicolumn{7}{|l|}{ Time (hours) } \\
\hline & 0 & 1 & 2 & 3 & 4 & 5 & 6 \\
\hline $\begin{array}{l}1 \\
2 \\
3 \\
4\end{array}$ & $\begin{array}{l}14.00(0.53) \\
13.97(0.48) \\
13.58(0.36) \\
14.08(0.53)\end{array}$ & $\begin{array}{l}13.26(0.61) \\
13.11(0.41) \\
12.85(0.56) \\
13.32(0.54)\end{array}$ & $\begin{array}{l}12.01(0.38) \\
11.85(0.61) \\
11.83(0.59) \\
12.92(0.39)\end{array}$ & $\begin{array}{l}11.94(0.49) \\
11.85(0.26) \\
11.83(0.21) \\
12.17(0.36)\end{array}$ & $\begin{array}{l}11.82(0.48) \\
11.51(0.23) \\
11.47(0.40) \\
11.84(0.37)\end{array}$ & $\begin{array}{l}11.68(0.34) \\
11.38(0.23) \\
11.33(0.54) \\
11.69(0.40)\end{array}$ & $\begin{array}{l}11.25(0.35) \\
11.19(0.34) \\
11.17(0.30) \\
11.46(0.34)\end{array}$ \\
\hline
\end{tabular}

by pure $\alpha_{1}$ antitrypsin abolished this difference (fig 1). When the samples were diluted to an equal degree with PBS, however, the effect on ciliary beat frequency, although less than that for the secretions alone ( $p<0.001$ for all values after one hour), was still clearly distinct from the inhibited samples $(p<$ 0.001 for all values after two hours). The results are summarised in figure 2 . The ciliary beat frequency fell from 13.75 (SEM $0 \cdot 17$ ) beats/s at zero time to $11.64(0 \cdot 13)$ beats/s at six hours in the $\alpha_{1}$ antitrypsin treated samples and from 14.08 $(0.16)$ beats/s to $8 \cdot 42(0 \cdot 19)$ beats/s in the PBS diluted preparations.

Sputum from these six patients collected after two weeks of antibiotic treatment had no detectable elastase activity and showed little effect on ciliary beat frequency, which fell from $13 \cdot 19(0 \cdot 20)$ beats/s

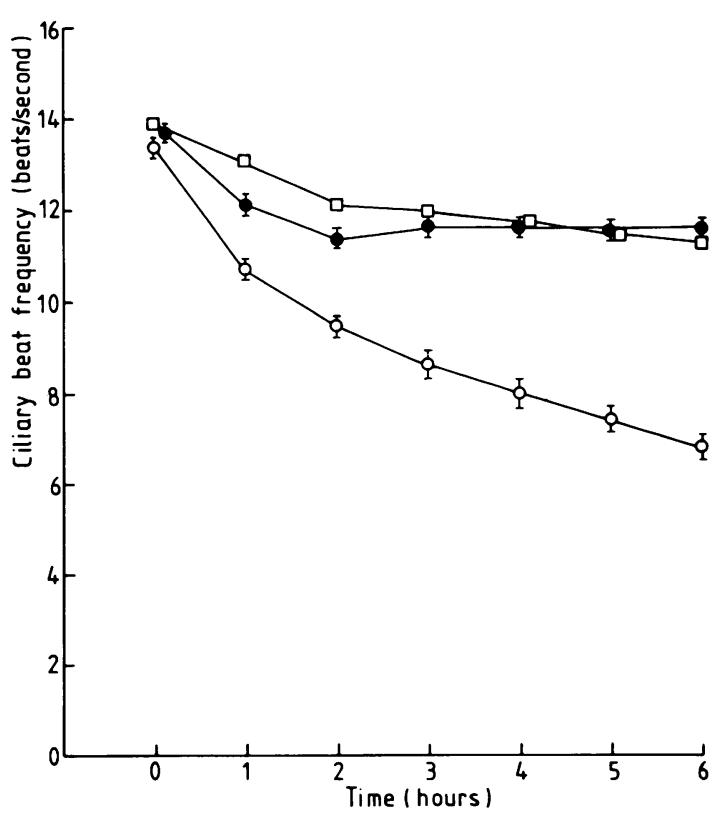

Fig 1 Ciliary beat frequency of human nasal epithelium in tissue culture medium alone ( $\square$ ), in elastase positive secretions from patients with bronchiectasis (O), and in secretions inhibited with pure human $\alpha_{1}$ antitrypsin (॰). Bar lines represent 1 standard error of the mean. to $10.49(0.18)$ beats/s over six hours. This was only significantly different from the control, consisting of tissue culture medium alone, after five and six hours $(\mathrm{p}<0.001)$ (fig 3).

\section{Discussion}

The present study shows that our results for nasal ciliary beat frequency in normal individuals are similar to those of other workers ${ }^{1213}$ and that cilia suspended in tissue culture medium continue to beat at a relatively constant rate for up to six hours, although there is a slight decrease in activity with time (table 2). This confirms the results of Dulfano et $a l,{ }^{15}$ who also found a relatively stable ciliary beat

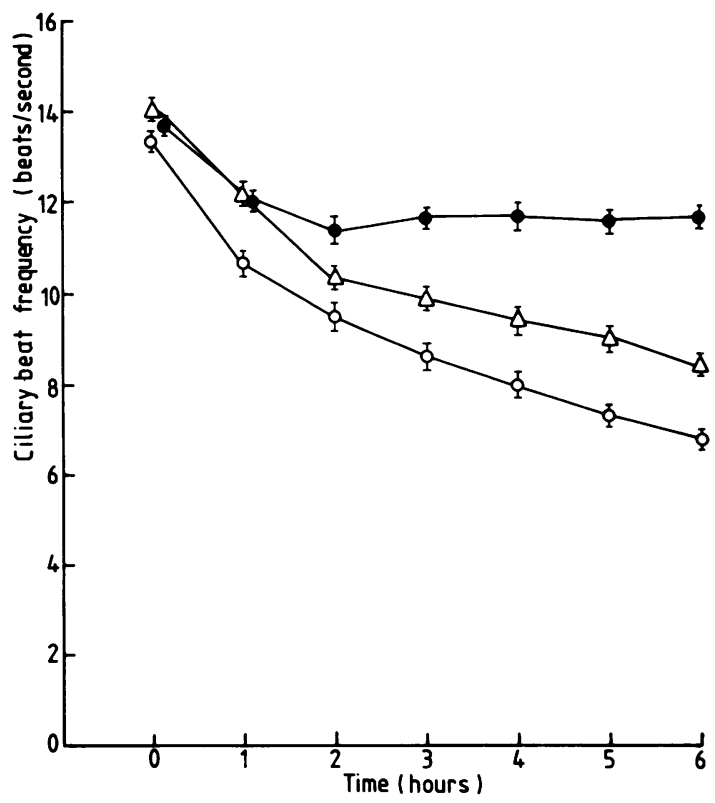

Fig 2 Effect of elastase positive secretions from patients with bronchiectasis (O) on ciliary beat frequency. The results of samples inhibited with pure human $\alpha_{1}$ antitrypsin (O) and those diluted to an equal degree with phosphate buffered saline $(\triangle)$ are also shown. Bar lines represent 1 standard error of the mean. 


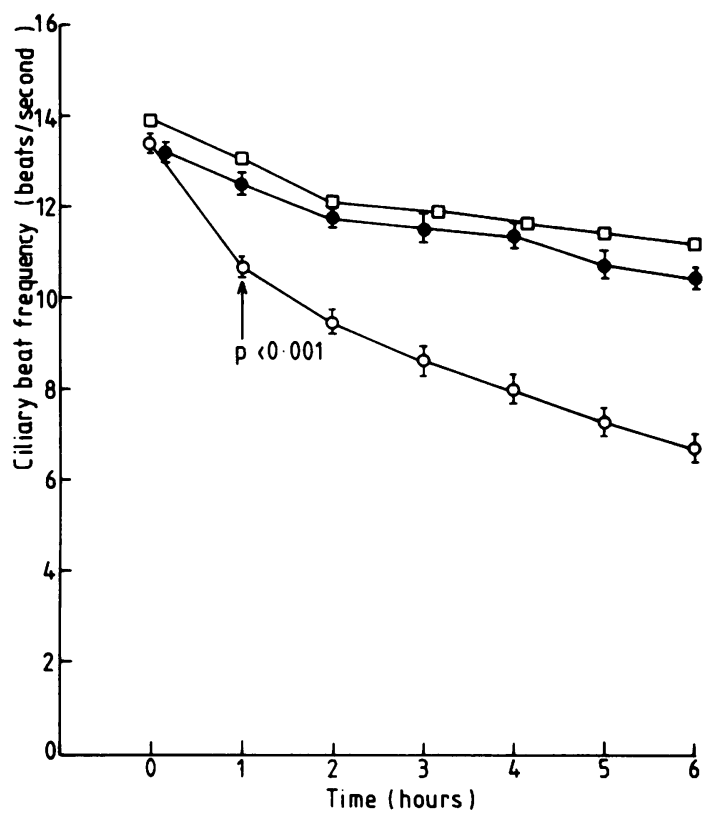

Fig 3 Effect of secretions from patients with bronchiectasis before $(O)$ and after (O) antibiotic treatment. The open squares ( $\square$ ) are the results for tissue culture medium alone.

frequency in preparations of bronchial epithelium studied for at least four hours.

Using this technique we showed that secretions from patients with purulent bronchiectasis had a detrimental effect on ciliary beat frequency in vitro (figs 1-3). This effect could be due to many possible ciliotoxic factors in the secretion. The $\mathrm{pH}$ of the samples is important since Luk et al ${ }^{16}$ have recently shown that such changes alone affect ciliary beat frequency; but this effect only occurs when the $\mathrm{pH}$ is outside the range $6 \cdot 5-9 \cdot 5$. The $\mathrm{pH}$ of all of our samples lay within this range, making this explanation unlikely. The effect might, however, be predicted from the elastase activity alone since such changes have been demonstrated by purified leucocyte elastase. ${ }^{8}$ To test this hypothesis we inhibited the elastase with pure $\alpha$, antitrypsin, confirming that the enzyme activity was due to a serine proteinase such as that from the neutrophil polymorph. The samples subsequently had no effect on ciliary beat frequency. The effect of elastase on the frequency is, however, dose dependent and, since the studies with $\alpha$ antitrypsin meant dilution of the samples with PBS, we repeated the experiments with PBS alone. The resultant effect on ciliary beat frequency was less than with the secretion alone but still distinct from that of the samples to which $\alpha_{1}$ antitrypsin had been added (figs 1 and 2). Whether the effect of bronchiectatic secretions on ciliary beat frequency is due to the elastase alone is uncertain since $\alpha_{1}$ antitrypsin is a broad spectrum inhibitor of serine proteinase, including cathepsin $G$ from the neutrophil polymorph and trypsin. Nevertheless, the effect on ciliary beat frequency of these secretions seems to be largely due to serine proteinase activity at least associated with the elastase detected. Furthermore, the reduction of the effect when the samples were diluted with PBS suggests a dose-response relationship, as seen by previous workers with the pure enzyme. ${ }^{8}$

After two weeks of treatment with amoxycillin the secretions from six of the patients showed no detectable elastase activity and had little effect on ciliary beat frequency (fig 3).

These results suggest that elastase or an associated serine proteinase is responsible for the ciliary dysfunction seen in vitro and thus could be partly responsible for decreased mucociliary clearance seen in patients with bronchiectasis. ${ }^{10}$ This effect, which has yet to be confirmed, would lead to greater retention of the secretions containing the potentially damaging enzyme and a vicious circle could be established. Further studies will be necessary to test this hypothesis and determine whether the manipulation of the secretions in vivo by antibiotics to remove elastase activity is associated with improvement in mucociliary function.

This study was supported by the General Hospital Bicentenary Appeal Fund, the Central Birmingham Health District Endowment Fund, the Rowbotham Bequest, and Bencard. One of us (LAS) wishes to thank Professor RC Curran for encouragement, advice, and laboratory facilities. We are also grateful to Dr J Newell for help with the photometer system, Mr SC Afford for technical assistance, and Miss A Wright for typing the manuscript.

\section{References}

' Eliasson R, Mossberg B, Camner P, Afzelius BA. The immotile-cilia syndrome. A congenital ciliary abnormality as an etiologic factor in chronic airway infections and male sterility. $N$ Engl J Med 1977;297: $1-6$.

${ }^{2}$ Sleigh MA. Primary ciliary dyskinesia. Lancet 1981 ;ii:476.

${ }^{3}$ Spock A, Heick MC, Cress H, Logan WC. Abnormal serum factor in patients with cystic fibrosis of the pancreas. Pediatr Res 1967;1:173-7.

${ }^{4}$ Conover JH, Conod EJ, Hirschhorn K. Ciliary dyskinesia factor in immunological and pulmonary disease. Lancet 1973;i: 1194.

5 Wilson GB, Fudenberg HH. Ciliary dyskinesia factors in cystic fibrosis and asthma. Nature 1977;266:463-4.

- Dulfano MJ, Luk CK, Beckage M, Wooten O. Ciliary 
inhibitory effects of asthma patients' sputum. Clin Sci 1982;63:393-6.

${ }^{7}$ Dulfano MJ, Luk CK. Sputum and ciliary inhibition in asthma. Thorax 1982;37:646-51.

${ }^{8}$ Tegner H, Ohlsson K, Toremalm NG, Von Mecklenburg C. Effect of human leucocyte enzymes on tracheal mucosa and its mucociliary activity. Rhinology 1979;17:199-206.

${ }^{9}$ Stockley RA, Hill SL, Morrison HM, Starkie CM. The elastolytic activity of sputum and its relationship to purulence and lung function in patients with bronchiectasis. Thorax 1984;39:408-13.

${ }^{10}$ Laurenço RV, Loddenkemper R, Carton RW. Patterns of distribution and clearance of aerosols in patients with bronchiectasis. Am Rev Respir Dis 1972;106:857-66.

$"$ Thurlbeck WM. Chronic airflow obstruction in lung dis- ease. Philadelphia: WB Saunders 1976:68-73.

12 Rutland J, Cole PJ. Non-invasive sampling of nasal cilia for measurement of beat frequency and study of ultrastructure. Lancet 1980;ii:564-5.

13 Rutland J, Griffin WM, Cole PJ. Human ciliary beat frequency in epithelium from intrathoracic and extrathoracic airways. Am Rev Respir Dis 1982;125: 100-5.

14 Stockley RA, Afford SC. The immunological assessment of $\alpha_{1}$-antitrypsin with reference to its function in bronchial secretions. Clin Sci 1983;65:373-81.

15 Dulfano MJ, Luk CK, Beckage M, Wooten O. Ciliary beat frequency in human respiratory explants. $A m$ Rev Respir Dis 1981;123:139-40.

${ }^{16}$ Luk CK, Dulfano MJ. Effect of pH, viscosity and ionicstrength changes on ciliary beating frequency of human bronchial explants. Clin Sci 1983;64:449-51. 